\title{
Value of Fecal Calprotectin Measurement During the Initial Period of Therapeutic Anti-Tubercular Trial
}

\author{
Hyeong Ho Jo', Eun Young Kim', Jin Tae Jung', Joong Goo Kwon', Eun Soo Kim², Hyun Seok Lee, Yoo Jin Lee, Kyeong Ok Kim", \\ Byung Ik Jang ${ }^{4}$, and the Crohn's and Colitis Association in Daegu-Gyeongbuk
}

${ }^{1}$ Department of Internal Medicine, Daegu Catholic University School of Medicine, Daegu, ${ }^{2}$ Department of Internal Medicine, School of Medicine, Kyungpook National University, Daegu, ${ }^{3}$ Department of Internal Medicine, Keimyung University School of Medicine, Daegu, ${ }^{4}$ Department of Internal Medicine, Yeungnam University College of Medicine, Daegu, Korea

Background/Aims: The diagnosis of intestinal tuberculosis (ITB) is often challenging. Therapeutic anti-tubercular trial (TATT) is sometimes used for the diagnosis of ITB. We aimed to evaluate the changing pattern of fecal calprotectin (FC) levels during TATT in patients with ITB.

Methods: A retrospective review was performed on the data of 39 patients who underwent TATT between September 2015 and November 2018 in five university hospitals in Daegu, South Korea. The analysis was performed for 33 patients with serial FC measurement reports.

Results: The mean age of the participants was 48.8 years. The final diagnosis of ITB was confirmed in 30 patients based on complete mucosal healing on follow-up colonoscopy performed after 2 months of TATT. Before starting TATT, the mean FC level of the ITB patients was $170.2 \mu \mathrm{g} / \mathrm{g}$ (range, 11.5-646.5). It dropped to $25.4 \mu \mathrm{g} / \mathrm{g}$ (range, 11.5-75.3) and then $23.3 \mu \mathrm{g} / \mathrm{g}$ (range, 11.5-172.2) after one and two months of TATT, respectively. The difference in mean FC before and one month after TATT was statistically significant $(p<0.001)$, and FC levels decreased to below $100 \mu \mathrm{g} / \mathrm{g}$ in all patients after one month of TATT.

Conclusions: All ITB patients showed FC decline after only 1 month of TATT, and this finding correlated with complete mucosal healing in the follow-up colonoscopy after 2 months of TATT. Clin Endosc 2022;55:256-262

Key Words: Diagnosis; Fecal calprotectin; Intestinal tuberculosis; Therapeutic anti-tubercular trial

\section{INTRODUCTION}

Although the number of patients with tuberculosis has decreased significantly compared to the past, South Korea still has the highest incidence and prevalence of tuberculosis compared to other countries in the Organisation for Economic Co-operation and Development (OECD). ${ }^{1}$ Consequential-

\footnotetext{
Received: January 25, 2021 Revised: July 10, 2021

Accepted: July 11, 2021

Correspondence: Eun Young Kim

Division of Gastroenterology, Department of Internal Medicine, Daegu Catholic University School of Medicine, 33 Duryugongwon-ro 17-gil, Nam-gu, Daegu 42472, Korea

Tel: +82-53-650-4092, Fax: +82-53-621-4487, E-mail: kimey@cu.ac.kr ORCID: https://orcid.org/0000-0003-3965-9964
}

(c) This is an Open Access article distributed under the terms of the Creative Commons Attribution Non-Commercial License (http://creativecommons.org/ licenses/by-nc/3.0) which permits unrestricted non-commercial use, distribution, and reproduction in any medium, provided the original work is properly cited. ly, intestinal tuberculosis (ITB) is not uncommon in South Korea.

Sometimes, it is difficult or tricky to diagnose and differentiate ITB from other diseases, especially Crohn's disease (CD). For the diagnosis of ITB, clinical features, colonoscopic findings, histologic examination, including Ziehl-Neelsen staining, culture, and polymerase chain reaction (PCR) tests, are extensively used. When ITB is suspected but there is still insufficient evidence to support its diagnosis, therapeutic anti-tubercular trial (TATT) is occasionally adopted. ${ }^{2}$ After TATT, the final diagnosis of ITB is accredited by confirming the healing of the lesions in a follow-up colonoscopy.

Calprotectin is a protein released by neutrophils. ${ }^{3}$ An increased amount of calprotectin is released into the stool by the migrated neutrophils if there is inflammation in the gastrointestinal tract. Fecal calprotectin (FC) concentration is known to correlate well with endoscopic disease activity and even more with the degree of histological inflammation in 
inflammatory bowel disease. ${ }^{3}$ However, FC in ITB has rarely been evaluated for its diagnostic significance or availability as an indicator for assessing disease activity. Considering that FC is a marker that is well proportional to the degree of intestinal inflammation, its level can also be expected to be highly correlated with the disease activity of ITB.

We aimed to evaluate the changes in FC levels in patients with a diagnosis of possible or probable ITB and attempted to determine the value of FC measurement during TATT.

\section{MATERIALS AND METHODS}

A retrospective review was performed for the TATT data of 39 patients treated from September 2015 to November 2018 at five university hospitals in Daegu, South Korea. Serial monthly FC level measurements were available for 33 patients. FC levels were measured using the fluorescence enzyme immunoassay method by Green Cross Laboratories, Yongin, Gyeonggi-do, Korea. Of the 33 patients, 30 were confirmed to have ITB based on the complete healing of the lesions in the follow-up colonoscopies performed two months after TATT. We analyzed the clinical and endoscopic characteristics and changes in the FC levels in these patients.

\section{Statistical analysis}

We conducted a descriptive analysis of the demographic and clinical characteristics as well as the colonoscopic findings of the patients and summarized the quantitative data by means, standard deviations, and qualitative data by frequencies and percentages. Statistical analysis was performed using IBM $^{\circledR}$ SPSS $^{\circledR}$ Statistics 25.0 (IBM; Armonk, NY, USA). After confirming that the FC levels do not follow a normal distribution by normality tests with Shapiro-Wilk test and Kolmogorov-Smirnov test, we analyzed the change in FC levels before and after TATT using the Wilcoxon signed-rank test.

\section{RESULTS}

\section{Clinical and colonoscopic characteristics}

The mean \pm standard deviation of the age of the patients was $48.8 \pm 11.3$ years. Twelve (36.4\%) patients were men. All of them had ulcerative lesions on the initial colonoscopy, and some of them showed pseudopolyps, patulous IC valve, or aphthous erosions. The number of the involved segments was $<4$ in most of the patients (97\%). The biopsy performed at the site of the ulcer showed faint suspicious granulomas in about 16 patients (48\%), but they were not the typical granulomas

Table 1. Clinical and Colonoscopic Characteristics

\begin{tabular}{|c|c|c|c|c|}
\hline Characteristics & $\begin{array}{l}\text { Overall } \\
(n=33)\end{array}$ & $\begin{array}{l}\text { Intestinal tuberculosis } \\
\qquad(n=30)\end{array}$ & $\begin{array}{l}\text { Crohn's disease } \\
\qquad(n=2)\end{array}$ & $\begin{array}{c}\text { Behcet's disease } \\
\qquad(n=1)\end{array}$ \\
\hline Age (year), mean $\pm \mathrm{SD}$ & $48.8 \pm 11.3$ & $49.7 \pm 9.9$ & $38 \pm 31.1$ & 44 \\
\hline Male, n (\%) & $12(36.4)$ & $10(33.4)$ & $1(50)$ & $1(100)$ \\
\hline IGRA (+), n (\%) & $28(84.8)$ & $27(90.0)$ & $0(0)$ & $1(100)$ \\
\hline Sequele of tuberculosis on chest X-ray, n (\%) & $4(12.1)$ & $4(13.3)$ & $0(0)$ & $0(0)$ \\
\hline \multicolumn{5}{|l|}{ Colonoscopic findings, $\mathrm{n}(\%)$} \\
\hline Patulous ileocecal valve & $15(45.5)$ & $15(50.0)$ & $0(0)$ & $0(0)$ \\
\hline Transverse ulcer & $14(42.4)$ & $14(46.7)$ & $0(0)$ & $0(0)$ \\
\hline Pseudopolyp & $16(48.5)$ & $16(53.3)$ & $0(0)$ & $0(0)$ \\
\hline Aphthous erosion & $10(30.3)$ & $8(26.7)$ & $2(100)$ & $0(0)$ \\
\hline Involvement $<4$ segments & $32(97.0)$ & $30(100)$ & $1(50.0)$ & $1(100)$ \\
\hline \multicolumn{5}{|l|}{ Colonoscopic biopsy findings, n (\%) } \\
\hline Granuloma with caseous necrosis $(+)$ & $0(0)$ & $0(0)$ & $0(0)$ & $0(0)$ \\
\hline $\mathrm{AFB}(+)$ & $0(0)$ & $0(0)$ & $0(0)$ & $0(0)$ \\
\hline Tbc-PCR (+) & $1(3.0)$ & $1(3.3)$ & $0(0)$ & $0(0)$ \\
\hline
\end{tabular}

AFB, acid-fast bacilli; IGRA, interferon-gamma release assays; SD, standard deviation; Tbc-PCR, polymerase chain reaction for Mycobacterium tuberculosis. 
with caseous necrosis. Acid-fast bacilli (AFB) staining of the obtained tissue was negative. The PCR for Mycobacterium tuberculosis (Tbc-PCR) results were negative in 32 patients but trace positive in one patient. AFB culture was later proven to be positive in two patients.

When colonoscopy was performed after two months of TATT, 30 patients showed complete healing of the lesions, which confirmed the diagnosis of ITB in these patients. For the other patients, two were later diagnosed with $\mathrm{CD}$ and one with Behcet's disease, based on clinical, radiological, and laboratory features.

The clinical and colonoscopic characteristics of the $33 \mathrm{pa}-$ tients included in the analysis are summarized in Table 1.

\section{Results of blood test}

In the blood tests for inflammatory biomarkers, the initial erythrocyte sedimentation rate (ESR) and C-reactive protein (CRP) were elevated in 8 (27\%) and $3(10 \%)$ patients, respectively (Fig. 1). Interferon-gamma release assay (IGRA) was negative in 5 (15\%) patients, including the 2 patients with CD. The Behçet's disease patient also had positive IGRA test results (Table 1).

\section{Changes in fecal calprotectin levels}

The mean FC level of the 30 ITB patients was 170.2 (range, 11.5-646.5) $\mu \mathrm{g} / \mathrm{g}$ at the start of TATT (Fig. 1). The mean FC level dropped to 25.4 (range, 11.5-75.3) $\mu \mathrm{g} / \mathrm{g}$ and 23.3 (range, 11.5-172.2) $\mu \mathrm{g} / \mathrm{g}$ after one and two months of TATT,
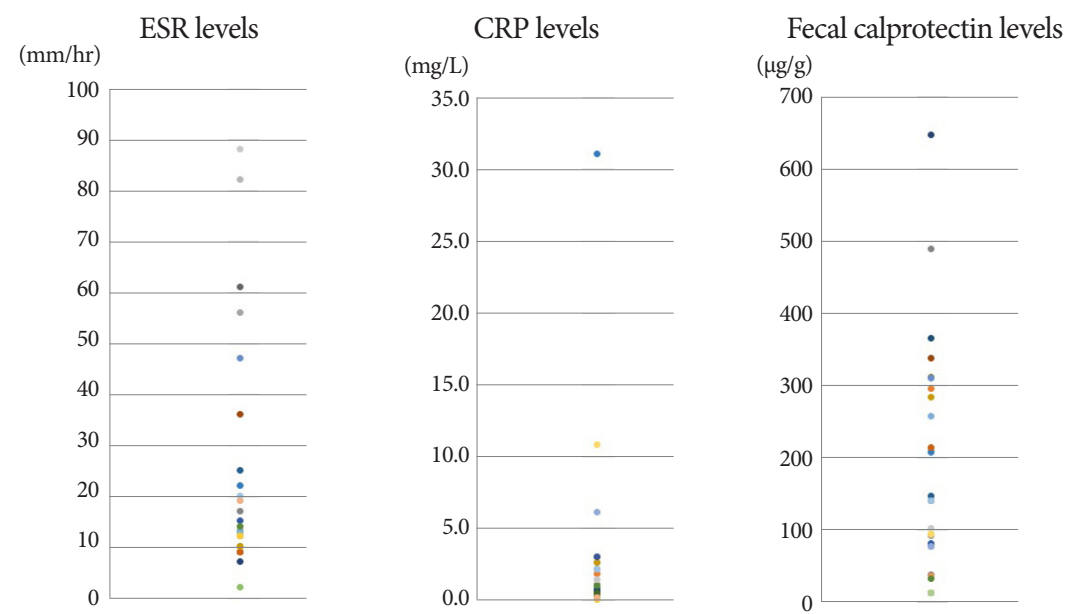

Fig. 1. Initial findings of inflammatory biomarkers in patients with intestinal tuberculosis. CRP, C-reactive protei; ESR, erythrocyte sedimentation rate.

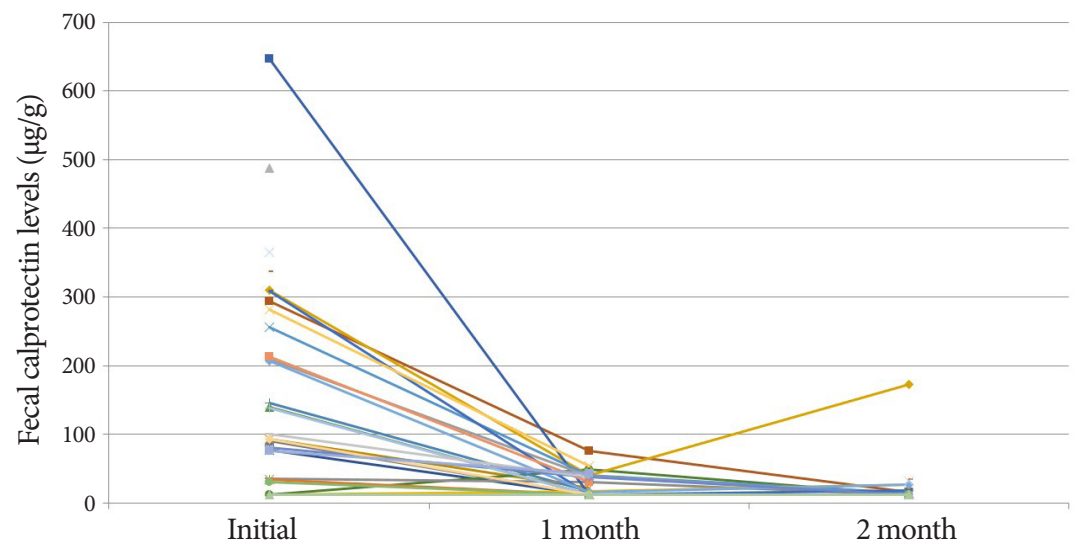

Fig. 2. Changes in fecal calprotectin levels after therapeutic anti-tuberculosis trial in individual patients with intestinal tuberculosis. 


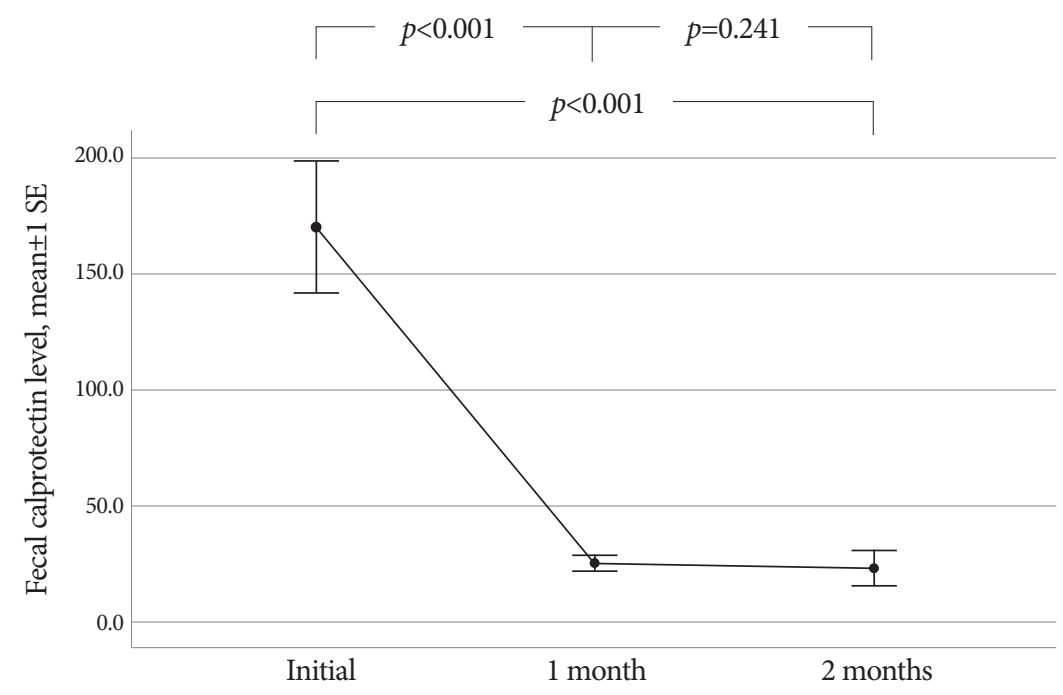

Fig. 3. Changes in the mean value of fecal calprotectin levels after therapeutic anti-tubercular trial in intestinal tuberculosis patients. SE, standard error.

Table 2. Changes of Fecal Calprotectin Levels

\begin{tabular}{lccc}
\hline \multicolumn{1}{c}{ Final diagnosis } & Initial & 1 month after TATT & 2 months after TATT \\
\hline Intestinal tuberculosis $(n=30), \mu \mathrm{g} / \mathrm{g}$, mean (range) & $170.2(11.5-646.5)$ & $25.4(11.5-75.3)$ & $23.3(11.5-172.2)$ \\
Crohn's disease (case 1), $\mu \mathrm{g} / \mathrm{g}$ & 580.1 & 1,515 & 182.4 \\
Crohn's disease (case 2), $\mu \mathrm{g} / \mathrm{g}$ & 2,000 & 2,000 & 2,000 \\
Behcet's disease (case 1), $\mu \mathrm{g} / \mathrm{g}$ & 138.6 & 24.5 & 220.9 \\
\hline
\end{tabular}

TATT, therapeutic anti-tubercular trial.

respectively, with all the patients showing less than $100 \mu \mathrm{g} / \mathrm{g}$ FC during follow up (Fig. 2). There was a statistically significant decrease in the mean FC level after one month of TATT $(p<0.001)$ (Fig. 3). On the other hand, the two patients who were confirmed to have $\mathrm{CD}$ showed no significant changes in FC levels between initial, 1 month, and 2 months after TATT (580.1/1515/182.4 $\mu \mathrm{g} / \mathrm{g}$, and 2000/2000/2000 $\mu \mathrm{g} / \mathrm{g}$, respectively). The patient who was later confirmed to have Behcet's disease also showed no significant changes in FC levels between initial, 1 month, and 2 months after TATT (138.6/24.5/220.9 $\mu \mathrm{g} / \mathrm{g}$, respectively) (Table 2).

\section{DISCUSSION}

Although ITB is not a rare disease in South Korea, its diagnosis is often challenging for physicians. ITB has a wide spec- trum of clinical manifestations and can mimic other diseases, such as CD. Correct diagnosis is important because it is a curable disease in contrast to $\mathrm{CD}$ that has at progressive relapsing course. As CD has had an increasing prevalence in South Korea, it has been emphasized as a differential diagnosis for ITB.

When ITB is present in the absence of pulmonary disease, diagnosis is confirmed if Ziehl-Nielsen staining of a colonoscopic biopsy specimen shows AFB or typical caseous granulomas or Mycobacterium tuberculosis is demonstrated in culture or Tbc-PCR of the biopsy specimen. However, the diagnostic sensitivity of AFB staining is $5.3-37.5 \%,{ }^{4 \cdot 6}$ the sensitivity of culture is $23-46 \%,{ }^{7,8}$ and the sensitivity of Tbc-PCR is $36.4-67.9 \%^{1,6,8-10}$ in ITB. All 30 ITB cases in this study showed negative AFB staining and negative Tbc-PCR results, except for one case of trace-positive Tbc-PCR results.

Serum markers, such as CRP and ESR, are also used for diagnosis, but their sensitivity and specificity are similarly low. 
In previous studies, elevated ESR was noted in $16-38 \%$ and elevated CRP levels in 50\% of ITB patients. ${ }^{11}$ In our study, the initial CRP levels were within the normal range in most ITB patients. The initial ESR was also in the normal range in more than half of the patients.

In this study, three ITB patients showed negative IGRA test results. In a previous study on the effectiveness of IGRA in the diagnosis of ITB, the sensitivity and specificity of the IGRA test were reported as 0.74 (95\% confidence interval [CI], 0.680.80 ) and 0.87 (95\% CI, 0.82-0.90), respectively. ${ }^{12}$ Advanced age (being over 65 years) and low peripheral lymphocyte counts are known to be common risk factors for false-negative IGRA results. ${ }^{13}$ Among the three IGRA negative ITB patients in our study, one patient was 72 years old and the other two patients' white blood cell counts were $4400 / \mu \mathrm{l}$ and $4470 / \mu \mathrm{l}$, respectively, with low lymphocyte fractions.

Endoscopic findings, such as involvement of fewer than four segments, transverse ulcers, pseudopolyps, scars, and patulous ileocecal value, are known to be the characteristic features of ITB. ${ }^{9}$ All ITB patients in our study showed less than four segment involvement, especially proximal colonic involvement, but a suggestive pattern of transverse ulcers was noted only in 14 cases $(42.4 \%)$. Half of the ITB patients showed a patulous ileocecal valve.

Due to the difficulties in the diagnosis of ITB, gastroenterologists are occasionally obliged to apply TATT for the diagnosis or differential diagnosis of ITB. The Asia-Pacific guidelines recommend TATT for 8-12 weeks in suspected patients due to the risk of disseminated tuberculosis if patients with ITB are misdiagnosed with $\mathrm{CD}$ and get immunosuppressive therapy. ${ }^{2}$ On the contrary, there also exists a risk that TATT may delay the diagnosis of $\mathrm{CD}$ and increase the risk of long-term complications, and that it may also lead to hepatotoxicity. ${ }^{1}$ In a recent report from Korea, $48 \%$ of patients with ITB required TATT for final diagnosis, and $18 \%$ of patients with $\mathrm{CD}$ received TATT initially. ${ }^{14}$

The fact that feces are in direct contact with the mucosa would be more conducive to the detection of intestinal inflammatory conditions by the measurement of fecal markers than by the measurement of biomarkers in serum. ${ }^{15,16}$ Among the biomarkers in feces, calprotectin is a calcium- and zinc-binding protein of the S- 100 protein family that was first isolated from blood leukocytes. ${ }^{17}$ Calprotectin accounts for $60 \%$ of the cytosolic protein in neutrophils. When cells are destroyed or activated, they are secreted to the outside of the cells, so it is a potential marker that is well proportional to the degree of intestinal inflammation. ${ }^{3}$ It has been reported that calprotectin in feces is highly resistant to degradation by intestinal pancreatic secretions, intestinal proteases, and bacterial degradation, and that it is stable for about a week at room temperature.
Since it is evenly distributed in the stool, it has been reported that it can be measured with a small amount of $5 \mathrm{~g}$ or less of feces. Therefore, many studies have been conducted regarding FC measurement in intestinal inflammation. ${ }^{15}$

Several studies have shown that FC is useful in the evaluation of digestive system inflammation as a biological marker reflecting the inflammatory state in the body. In particular, FC levels are highly correlated with the disease activity of inflammatory bowel disease, ${ }^{5}$ which can be useful in discriminating between irritable bowel syndrome and inflammatory bowel disease, evaluating the disease activity of inflammatory bowel disease, evaluating response after treatment, and predicting recurrence after medical or surgical remission. ${ }^{18}$

However, studies on the value of FC in the diagnosis and treatment of ITB are insufficient. One recent study attempted to determine whether serial measurements of fecal calprotectin during TATT can help in dustiguishing ITB from $\mathrm{CD} .{ }^{19} \mathrm{In}$ that study, the diagnosis of ITB was confirmed by demonstrating the colonoscopic findings of mucosal healing at 2 months of TATT. They reported that 28 ITB patients showed a statistically significant decrease in FC levels at two months and six months of TATT compared to one lymphoma and eight CD patients. FC levels of ITB patients at baseline, 2 months, and 6 months were $216 \mu \mathrm{g} / \mathrm{g}, 43 \mu \mathrm{g} / \mathrm{g}$, and $26 \mu \mathrm{g} / \mathrm{g}$, respectively. That is quite similar to our study results of $170.2 \mu \mathrm{g} / \mathrm{g}$ and $23.3 \mu \mathrm{g} / \mathrm{g}$ at baseline and 2 months of TATT, respectively. Our study additionally showed that the FC level had significantly decreased only 1 month after TATT in all ITB patients, and this finding correlated well with complete mucosal healing in follow-up colonoscopy after two months of TATT. This indicates that a significant decrease in the FC level after one month of TATT can suggest a significant decrease in FC level and colonoscopic mucosal healing after two months of TATT, confirming the diagnosis of ITB by inference.

When TATT is adopted in an ambiguous case, the appropriate duration before response assessment with follow-up colonoscopy is not clearly defined. Generally, endoscopic reevaluation is performed in 2-3 months of TATT. Compared to colonoscopy, which is invasive, requires preparation, and is a relatively expensive test, FC measurement is a non-invasive, simple, and inexpensive test. If significant FC decrement at one month of TATT can be used as a biomarker for the diagnosis of ITB, it would be important beneficial information to patients under TATT and gastroenterologists who subscribe to TATT.

Since there is no standardized cut-off value of FC levels for the diagnosis of each disease, there is a limitation in trying to show the diagnostic significance of FC measurement in ITB. According a previous study, the cut-off value varied from 50 to $250 \mu \mathrm{g} / \mathrm{g}$ in the diagnosis or evaluation of disease activity in in- 
flammatory bowel disease. ${ }^{20}$ In our study, the mean FC level of the ITB patients was $170.2 \mu \mathrm{g} / \mathrm{g}$ (range, 11.5-646.5) at baseline, and 16 (53.3\%) had FC levels above $100 \mu \mathrm{g} / \mathrm{g}$, while $22(73.3 \%)$ had FC levels above $50 \mu \mathrm{g} / \mathrm{g}$. The mean FC level was $25.4 \mu \mathrm{g} /$ $\mathrm{g}$ (range, 11.5-75.3) at one month after TATT, and all patients showed FC levels below $100 \mu \mathrm{g} / \mathrm{g}$. Considering that the number of the enrolled cases in this study was quite small, it seems difficult to draw the cut-off level to define a good response to TATT after one month from the results of this study.

In addition, FC levels may be measured at various concentrations per day, depending on the time of measurement. If the period between bowel movements is long, the level may be high; therefore, it is recommended that the first test be performed in the morning. Unfortunately, due to the retrospective nature of our study, we could not determine when the samples were collected from each patient. Studies have shown that taking drugs, such as nonsteroidal anti-inflammatory drugs, proton pump inhibitors, and aspirin, increases fecal calprotectin levels. ${ }^{21,22}$ Regrettably, we could not collect medication history from all the enrolled patients.

In conclusion, the mean FC level of patients with ITB dropped significantly after one month of TATT. A drop of the FC level below $100 \mu \mathrm{g} / \mathrm{g}$ after one month of TATT might be a helpful clue in diagnosing ITB. However, the initial FC level in patients with ITB had a wide range, so we must consider which patients may benefit from the serial measurement of FC levels. Further research is needed to confirm the correlation between colonoscopic disease activity and FC levels in patients with ITB. This may allow us to select the right patients whose FC measurements and serial monitoring can assist in making a definitive diagnosis when the initial colonoscopy findings are ambiguous. The role of FC measurement in the differential diagnosis of CD and ITB during TATT should also be studied in a larger number of patients.

\section{Conflicts of Interest}

Eun Young Kim is currently serving as an editor emeritus in Clinical Endoscopy; however, she had not involved in the peer reviewer selection, evaluation, or decision process of this article. The other authors have no potential conflicts of interest.

Funding

This work was supported by a grant from the Research Institute of Medical Science, Daegu Catholic University (2015).

\section{Acknowledgments}

We gratefully acknowledge the support of the members of the Crohn's and Colitis Association in Daegu-Gyeongbuk (CCAiD), especially Sungkook Kim and Jun Heo.

\section{Author Contributions}

Conceptualization: Eun Young Kim, Byung Ik Jang
Data curation: Eun Soo Kim, Hyun Seok Lee, Yoo Jin Lee, Kyeong Ok Kim

Funding acquisition: EYK

Methodology: Jin Tae Jung, Joong Goo Kwon

Supervision: EYK

Writing-original draft: Hyeong Ho Jo

Writing-review \& editing: HHJ, EYK

\section{ORCID}

Hyeong Ho Jo

Eun Young Kim

Jin Tae Jung

Joong Goo Kwon

Eun Soo Kim

Hyun Seok Lee

Yoo Jin Lee

Kyeong Ok Kim

Byung Ik Jang https://orcid.org/0000-0002-4950-5435 https://orcid.org/0000-0003-3965-9964 https://orcid.org/0000-0002-2238-5306 https://orcid.org/0000-0003-3392-785X https://orcid.org/0000-0003-0806-9136 https://orcid.org/0000-0001-9288-0321 https://orcid.org/0000-0003-1799-0146 https://orcid.org/0000-0001-5799-7436 https://orcid.org/0000-0002-3037-9272

\section{REFERENCES}

1. Jin XJ, Kim JM, Kim HK, et al. Histopathology and TB-PCR kit analysis in differentiating the diagnosis of intestinal tuberculosis and Crohn's disease. World J Gastroenterol 2010;16:2496-2503.

2. Ooi CJ, Makharia GK, Hilmi I, et al. Asia Pacific Consensus Statements on Crohn's disease. Part 1: definition, diagnosis, and epidemiology: (asia pacific Crohn's disease consensus--Part 1). J Gastroenterol Hepatol 2016;31:45-55.

3. Pathirana WGW, Chubb SP, Gillett MJ, Vasikaran SD. Faecal calprotectin. Clin Biochem Rev 2018;39:77-90.

4. Dutta AK, Sahu MK, Gangadharan SK, Chacko A. Distinguishing Crohn's disease from intestinal tuberculosis--a prospective study. Trop Gastroenterol 2011;32:204-209.

5. Li Y, Zhang L, Liu X, et al. The role of in vitro interferon $\gamma$-release assay in differentiating intestinal tuberculosis from Crohn's disease in China. J Crohns Colitis 2012;6:317-323.

6. Lei Y, Yi FM, Zhao J, et al. Utility of in vitro interferon- $\gamma$ release assay in differential diagnosis between intestinal tuberculosis and Crohn's disease. J Dig Dis 2013;14:68-75.

7. Ramadass B, Chittaranjan S, Subramanian V, Ramakrishna BS. Fecal polymerase chain reaction for Mycobacterium tuberculosis IS6110 to distinguish Crohn's disease from intestinal tuberculosis. Indian J Gastroenterol 2010;29:152-156.

8. Amarapurkar DN, Patel ND, Rane PS. Diagnosis of Crohn's disease in India where tuberculosis is widely prevalent. World J Gastroenterol 2008;14:741-746.

9. Makharia GK, Srivastava S, Das P, et al. Clinical, endoscopic, and histological differentiations between Crohn's disease and intestinal tuberculosis. Am J Gastroenterol 2010;105:642-651.

10. Fei BY, Lv HX, Zheng WH. Fluorescent quantitative PCR of Mycobacterium tuberculosis for differentiating intestinal tuberculosis from Crohn's disease. Braz J Med Biol Res 2014;47:166-170.

11. Kim YS, Kim YH, Lee KM, Kim JS, Park YS, IBD Study Group of the Korean Association of the Study of Intestinal Diseases. Diagnostic guideline of intestinal tuberculosis. Korean J Gastroenterol 2009;53:177186.

12. Chen W, Fan JH, Luo W, Peng P, Su SB. Effectiveness of interferon-gamma release assays for differentiating intestinal tuberculosis from Crohn's disease: a meta-analysis. World J Gastroenterol 2013;19:8133-8140.

13. Yamasue M, Komiya K, Usagawa Y, et al. Factors associated with false negative interferon- $\gamma$ release assay results in patients with tuberculosis: a systematic review with meta-analysis. Sci Rep 2020;10:1607. 
14. Seo H, Lee S, So H, et al. Temporal trends in the misdiagnosis rates between Crohn's disease and intestinal tuberculosis. World J Gastroenterol 2017;23:6306-6314.

15. Lee KM. Fecal biomarkers in inflammatory bowel disease. Intest Res 2013;11:73-78.

16. Konikoff MR, Denson LA. Role of fecal calprotectin as a biomarker of intestinal inflammation in inflammatory bowel disease. Inflamm Bowel Dis 2006;12:524-534

17. Lee YW, Lee KM, Lee JM, et al. The usefulness of fecal calprotectin in assessing inflammatory bowel disease activity. Korean J Intern Med 2019;34:72-80

18. Mumolo MG, Bertani L, Ceccarelli L, et al. From bench to bedside: fecal calprotectin in inflammatory bowel diseases clinical setting. World J Gastroenterol 2018;24:3681-3694.
19. Sharma V, Verma S, Kumar-M P, et al. Serial measurements of faecal calprotectin may discriminate intestinal tuberculosis and Crohn's disease in patients started on antitubercular therapy. Eur J Gastroenterol Hepatol 2021;33:334-338.

20. Desai D, Faubion WA, Sandborn WJ. Review article: biological activity markers in inflammatory bowel disease. Aliment Pharmacol Ther 2007;25:247-255

21. Lasson A, Stotzer PO, Öhman L, Isaksson S, Sapnara M, Strid H. The intra-individual variability of faecal calprotectin: a prospective study in patients with active ulcerative colitis. J Crohns Colitis 2015;9:26-32.

22. Higuchi K, Umegaki E, Watanabe T, et al. Present status and strategy of NSAIDs-induced small bowel injury. J Gastroenterol 2009;44:879-888. 